\title{
Balloon expandable transcatheter aortic valve for degenerative prior prosthetic valve dysfunction
}

\author{
Stephanie Tom, Emily Perdoncin, Kendra J. Grubb \\ Division of Cardiothoracic Surgery, Structural Heart and Valve Center, Emory University School of Medicine, Atlanta, GA, USA \\ Correspondence to: Kendra J. Grubb, MD, MHA, FACC. Associate Professor of Surgery, Surgical Director, Division of Cardiovascular Surgery, \\ Structural Heart and Valve Center, Emory University School of Medicine, 550 Peachtree Street NE, Davis-Fischer, Atlanta, GA 30308, USA. \\ Email: kjgrubb@emory.edu.
}

Submitted Apr 03, 2021. Accepted for publication Jun 25, 2021.

doi: 10.21037/acs-2021-tviv-12

View this article at: https://dx.doi.org/10.21037/acs-2021-tviv-12

Bioprosthetic surgical aortic valve replacement (SAVR) has increased dramatically over the past decade as patients seek to avoid lifelong anticoagulation (1). The trend has been fortified by the assurance of a future percutaneous treatment with a transcatheter aortic valve replacement (TAVR) within the existing SAVR, dubbed valve-in-valve (ViV) (2). The implications are most significant for younger, lowrisk patients whose life-expectancy will exceed that of the initial SAVR valve and may in fact exceed the ViV-TAVR, as all current bioprosthetic valves will eventually fail. TAVR as the initial valve in young patients further complicates decision making as long-term durability data is lacking (3-5). However, both ViV-TAVR and TAVR-in-TAVR (redoTAVR) are now viable options with improved short-term morbidity and mortality, when compared to redo-SAVR for higher risk patients (2).

Results from the PARTNER (Placement of Aortic Transcatheter Valves) II registry are encouraging for $\mathrm{ViV}$ TAVR with SAPIEN XT (Edwards Lifesciences, Irvine, CA, USA) balloon expandable valve (BEV), demonstrating sustained performance at three years with no change in gradients, effective orifice area, or aortic regurgitation, as well as improvement in quality of life and functional status (6). However, patients were high-risk and treated with only 23 or $26 \mathrm{~mm}$ Sapien XT valves, and SAVR valves $<21 \mathrm{~mm}$ were excluded.

The appropriate valve for a ViV-TAVR requires special attention to individual patient anatomy, as assessed by computed tomography angiography (CTA). Measuring the SAVR true internal diameter for TAVR valve sizing, and diameters of the sinotubular junction (STJ) as well as coronary heights for coronary obstruction risk, assists in determining ViV feasibility. The SAVR leaflet tissue creates a continuous skirt above the annulus when the TAVR is implanted and can result in sinus sequestration and coronary obstruction. A valve-to-coronary distance of $4 \mathrm{~mm}$ determines feasibility of $\mathrm{ViV}$ to avoid a catastrophic complication. The height of the BEV frame and intraannular position are favorable in this regard as the TAVR lands within or below the SAVR leaflets. However, the cylindrical frame of the BEV causes the SAVR leaflets to open fully, making future coronary access potentially challenging. Coronary access after $\mathrm{ViV}$ is a concern that applies to all transcatheter heart valve (THV) designs and the BEV may hold an advantage over the self-expanding valve (SEV), with regards to coronary access and risk for coronary obstruction during ViV-TAVR or future redoTAVR procedures. While using a SEV for ViV procedures may allow for retrieval or repositioning if there is evidence of impending coronary obstruction, the leaflets of the supra-annular SEV will often reach the STJ, thus making coronary access challenging and likely prohibiting a future redo-TAVR. In CTA analysis, coronary arteries originated below the top of the neo-skirt in $90 \%$ of SEV first cases, compared with $67 \%$ of BEVs, which may be compounded when the THV is within a SAVR frame. Further, the risk for technically impossible coronary access has been estimated at $27 \%$ for SEV and $10 \%$ for BEV (7). Leaflet modification strategies have been employed, such as electrosurgical techniques (BASILICA), in order to split the leaflets and increase coronary flow, but the technique is not yet widely adopted. Currently, there is no percutaneous leaflet removal strategy available for failed SAVR valves that mimics surgical leaflet excision. This has important implications for 
young patients, who may require three valves during their lifetime, and this further supports the use of $\mathrm{ViV}$ only in high-risk re-operative SAVR patients.

Although the BEV's low frame height has advantages with respect to coronary access, the position within an existing SAVR may come at the detriment of hemodynamics and long-term durability. The BEV intra-annular design has been associated with inferior hemodynamics compared with the supra-annular SEV. The Valve-in-Valve International Data Registry (VIVID) found elevated post-procedural gradients, defined as mean gradients $>20 \mathrm{mmHg}$, more common after BEV ViV than SEV ViV (40\% vs. $21.3 \%$, $\mathrm{P}<0.0001)$. Furthermore, $\mathrm{BEV}$ also appeared to perform poorly in small surgical valves (ID $<21 \mathrm{~mm}$ ), with higher rates of elevated post-procedural gradients when compared to $\operatorname{SEV~(58.8\% ~vs.~20\% ,~} \mathrm{P}=0.005)$ at one year follow-up (8). While these findings did not necessarily translate to obvious mortality differences, the long-term clinical implications are worrisome (9).

When assessing long-term outcomes, pre-existing SAVR patient prosthesis mismatch (PPM) was an independent predictor for re-intervention after $\mathrm{ViV}$ and was associated with decreased patient survival, demonstrating the need for careful valve selection at the time of the initial surgery as well as subsequent $\mathrm{ViV}(10)$. The poor hemodynamic results of BEV in small SAVR valves with pre-existing PPM, high residual gradient and increased need for re-intervention after $\mathrm{ViV}$ with BEV, cumulatively suggests the intra-annular valve may not be the right choice for these patients. Often THVs fail to fully expand in ViV procedures. Although elevated gradients can be seen in all $\mathrm{ViV}$ procedures, the $\mathrm{BEV}$ intra-annular design with leaflets constrained within the SAVR, can result in leaflet dysfunction which may increase the risk of thrombosis and earlier structural valve deterioration. Under-expanded THVs can be optimized by balloon fracture of the SAVR frame, resulting in lower gradients and potentially improved long-term durability. Due to lack of guidelines for anticoagulation post$\mathrm{ViV}$, many centers opt for six to twelve months of oral anticoagulation (warfarin or a novel oral anticoagulant such as apixaban) in addition to aspirin antiplatelet therapy.

In summary, the choice of THV for $\mathrm{ViV}$ procedures has long-term implications for hemodynamics and coronary access. Heart teams must carefully weigh the advantages and disadvantages of each THV. If the last 20 years of TAVR development can predict future device development, newer devices and adjunctive procedures will be invented to solve these issues. While we await these solutions, we must counsel patients on the lifetime management of aortic stenosis, presenting an anatomically optimized strategy not only for the immediate valve but also accounting for patients who may have more than 30 years to live and potentially require three valves. While SEV may be appropriate in patients with small surgical valves at risk for PPM, an optimized BEV with leaflet modification and fracture of the SAVR can provide excellent hemodynamics with superior coronary access and may therefore be the most appropriate choice for younger patients, who will most likely require an additional valve procedure during their lifetime.

\section{Acknowledgments}

Funding: None.

\section{Footnote}

Conflicts of Interest: KJG is a consultant for Edwards Lifesciences and Medtronic; national PI for Medtronic. The other authors have no conflicts of interest to declare.

Open Access Statement: This is an Open Access article distributed in accordance with the Creative Commons Attribution-NonCommercial-NoDerivs 4.0 International License (CC BY-NC-ND 4.0), which permits the noncommercial replication and distribution of the article with the strict proviso that no changes or edits are made and the original work is properly cited (including links to both the formal publication through the relevant DOI and the license). See: https://creativecommons.org/licenses/by-nc-nd/4.0/.

\section{References}

1. Alkhouli M, Alqahtani F, Kawsara A, et al. National Trends in Mechanical Valve Replacement in Patients Aged 50 to 70 Years. J Am Coll Cardiol 2020;76:2687-8.

2. Tam DY, Dharma C, Rocha RV, et al. Transcatheter ViV Versus Redo Surgical AVR for the Management of Failed Biological Prosthesis: Early and Late Outcomes in a Propensity-Matched Cohort. JACC Cardiovasc Interv 2020;13:765-74.

3. Leon MB, Mack MJ, Hahn RT, et al. Outcomes 2 Years After Transcatheter Aortic Valve Replacement in Patients at Low Surgical Risk. J Am Coll Cardiol 2021;77:1149-61.

4. Popma JJ, Deeb GM, Yakubov SJ, et al. Transcatheter Aortic-Valve Replacement with a Self-Expanding Valve in 
Low-Risk Patients. N Engl J Med 2019;380:1706-15.

5. Søndergaard L, Ihlemann N, Capodanno D, et al.

Durability of Transcatheter and Surgical Bioprosthetic

Aortic Valves in Patients at Lower Surgical Risk. J Am Coll Cardiol 2019;73:546-53.

6. Webb JG, Murdoch DJ, Alu MC, et al. 3-Year Outcomes After Valve-in-Valve Transcatheter Aortic Valve Replacement for Degenerated Bioprostheses: The PARTNER 2 Registry. J Am Coll Cardiol 2019;73:2647-55.

7. De Backer O, Landes U, Fuchs A, et al. Coronary Access After TAVR-in-TAVR as Evaluated by Multidetector Computed Tomography. JACC Cardiovasc Interv
2020;13:2528-38.

8. Dvir D, Webb J, Brecker S, et al. Transcatheter aortic valve replacement for degenerative bioprosthetic surgical valves: results from the global valve-in-valve registry. Circulation 2012;126:2335-44.

9. Lee HA, Chou AH, Wu VC, et al. Balloon-expandable versus self-expanding transcatheter aortic valve replacement for bioprosthetic dysfunction: A systematic review and meta-analysis. PLoS One 2020;15:e0233894.

10. Bleiziffer S, Simonato M, Webb JG, et al. Long-term outcomes after transcatheter aortic valve implantation in failed bioprosthetic valves. Eur Heart J 2020;41:2731-42.

Cite this article as: Tom S, Perdoncin E, Grubb KJ. Balloon expandable transcatheter aortic valve for degenerative prior prosthetic valve dysfunction. Ann Cardiothorac Surg 2021;10(5):686-688. doi: 10.21037/acs-2021-tviv-12 